\title{
EVALUACIÓN DE LA APTITUD ÉTICO-PROFESIONAL EN ESTUDIANTES EN PRÁCTICAS EN EDUCACIÓN FÍSICA EN LA UNIVERSIDAD DE COÍMBRA: UNA PRÁCTICA INTERESANTE
}

Evaluation of the Ethical-Professional Attitude in trainees of Physical Education of the University of Coimbra: an interesting practice

\section{Avaliação da Atitude Ético-Profissional em estagiários de Educação Física da Universidade de Coimbra: uma prática interessante}

\section{Elsa Ribeiro-Silva (1)}

\begin{abstract}
(1) Faculdade de Ciências do Desporto e Educação Física da Universidade de Coimbra, Portugal. Teléfono: +351 912996572. Correo electrónico: elsasilva@fcdef.uc.pt
\end{abstract}

\section{Resumen}

Este texto consiste en la presentación de las prácticas de evaluación utilizadas en el Practicum en Educación Física en la Facultad de Ciencias del Deporte y Educación Física de la Universidad de Coímbra, en respecto a la Dimensión Actitud ÉticoProfesional. Con este se pretende dar a conocer las dichas prácticas, considerándolas un ejemplo interesante para compartir con la sociedad científica. En el texto describimos el proceso de evaluación donde el alumnado en prácticas está integrado y presentamos el respectivo referencial de la referida dimensión, lo cual se estructura en diez grandes competencias a desarrollar, concretadas en descriptores por cada nivel de evaluación: Maestría (18-20 puntos), Habilidad (14-17 puntos), y Aprendizaje (10-13 puntos).

Palabras clave: Prácticas de Evaluación; Actitud Ético-Profesional; Formación Inicial; Educación Física

\begin{abstract}
This text consists of the presentation of the evaluation practices followed in the Teacher Training Period in Physical Education at the Faculty of Sports Sciences and Physical Education of the University of Coimbra, and it intends to make known the same practices, considering them an interesting example to share with scientific society. In this paper we describe the evaluation process in which trainees are integrated and present their respective framework, which is structured in four major dimensions of evaluation: i)Teaching-learning activities; ii) School Organization and Management


Activities; iii) Activities of Projects and Partnerships and iv) Professional Ethics, with emphasis on the fact that all the criteria of all the parameters of all dimensions of evaluation are operationalized in descriptors, thus orienting both trainees to what is expected of them in each level of evaluation: Mastery (18-20 values), Proficiency (1417 values) and Learning (10-13 values), as well as to the supervisors in their formative and summative evaluation process.

Keywords: Teacher Training Period; Physical Education; Evaluation practices; Evaluation framework

\section{Resumo}

Este texto consiste na apresentação das práticas de avaliação seguidas no Estágio Pedagógico em Educação Física na Faculdade de Ciências do Desporto e Educação Física da Universidade de Coimbra, no que se refere à Dimensão Atitude ÉticoProfissional. Com este pretende-se dar a conhecer aquelas mesmas práticas, considerando-as um exemplo interessante a partilhar com a sociedade científica. Neste descrevemos o processo de avaliação em que os estagiários estão integrados e apresentamos o respetivo referencial da referida dimensão, o qual está estruturado em dez grandes competências a desenvolver, operacionalizadas em descritores por cada um dos níveis de avaliação: Mestria (18-20 valores), Proficiência (14-17 valores) e Aprendizagem (10-13valores).

Palavras-chave: Práticas de Avaliação; Atitude Ético-Profissional; Formação Inicial; Educação Física

\section{Introducción}

Este trabajo pretende dar a conocer la forma como ha sido superada la dificultad de evaluar la Dimensión Actitud Ético-Profesional, en el Prácticum del Máster en Profesorado de Educación Física en Enseñanza Obligatoria y Bachillerato (MPEFEOB) de la Facultad de Ciencias del Deporte y Educación Física de la Universidad de Coímbra (FCDEFUC), Portugal.

En el MPEFEOB se considera, según Crisol y Romero (2014), que la formación ética debe estar incluida en el currículo de la formación universitaria como un componente sistemático dentro de la formación pedagógica, donde los aspectos cognitivos, morales 
y las habilidades técnicas deben fusionarse al objeto de adquirir una adecuada profesionalidad, razón por la cual aquella dimensión es transversal y decisiva en la evaluación del Practicum.

\section{Contextualización}

Esta experiencia se refiere al proceso evaluativo del Estágio Pedagógico (Prácticum) del Máster en Profesorado de Educación Física en Enseñanza Obligatoria y Bachillerato de la FCDEFUC, con lo cual se busca promocionar una preparación especializada en la aplicación de conocimientos en contextos multidisciplinares de intervención profesional en la enseñanza secundaria obligatoria y bachillerato (Ribeiro-Silva, Fachada, Nobre, 2018).

El Prácticum es una asignatura del plan de estudios del dicho máster, que cuenta con cerca de 50 estudiantes al año, y tiene como función final la profesionalización de nuevos docentes a través de un proceso de práctica profesional a lo largo de un año lectivo, diariamente acompañada de un tutor. Con objeto de volver claro y participativo el proceso de evaluación en el cual los alumnos en prácticas estarán integrados, confeccionamos anualmente documentación actualizada y completa sobre el proceso evaluativo, teniendo previsto tiempos de discusión conjunta entre los supervisores y el alumnado, para que estos tomen conocimiento, pero, sobre todo, para que puedan presentar propuestas pertinentes en relación a ese proceso.

La evaluación del Prácticum se basa en 4 grandes dimensiones, correspondiendo la cuarta a la Actitud Ético-Profesional, que es transversal a las demás y se presenta en diez competencias/parámetros, a saber:

1. Compromiso con los aprendizajes de los alumnos

2. Compromiso con sus propios aprendizajes

3. Conocimientos generales y específicos

4. Autoformación y desarrollo profesional

5. Disponibilidad para los alumnos y para la escuela

6. Trabajo en equipo

7. Capacidad de iniciativa y responsabilidad

8. Innovación de las prácticas pedagógicas y documentales

9. Análisis crítica y reflexiva

10. Conducta personal

Evaluación de la aptitud ético-profesional en estudiantes en prácticas en Educación Física en la 
Estos parámetros han sido construidos teniendo como base los objetivos de formación del máster y del Perfil General de Desempeño Profesional de los Profesores de la Enseñanza Secundaria Obligatoria y Bachillerato (Decreto Ley 240/2001, de 17 de agosto, del Gobierno de Portugal). Considerando que la ética y la profesionalidad docente son los pilares de la actuación del futuro profesor, y que se revelan constantemente en el cuadro de desempeño diario del alumno en prácticas, las competencias/criterios de esta Dimensión de evaluación del Prácticum vienen estructurados en tres niveles de su desempeño: Aprendizaje (10 a 13 puntos), Habilidad (14 a 17 puntos), y Maestría (18 a 20 puntos), donde se ha desarrollado para cada uno de ellos, descriptores adaptados a los 3 niveles evaluativos.

En términos de clasificación final, el alumnado en prácticas solo aprobará la asignatura cuando obtenga valores positivos en la totalidad de sus criterios de evaluación, así como, solo podrá obtener una nota final igual o superior a 18 valores (en un máximo de 20 valores) siempre y cuando adquiera ese mismo nivel máximo en la Dimensión Actitud Ético-Profesional.

\section{Diseño y desarrollo}

Teniendo como base este referencial, los supervisores de la facultad, en conjunto con los de la escuela, evalúan, tanto cuantitativamente como cualitativamente, cada uno de sus alumnos en prácticas, una vez consultada con estos su autoevaluación en relación a esta dimensión. Esta autoevaluación consiste en la realización, por el alumno, de un informe auto-reflexivo sobre el trabajo hecho a lo largo del curso, incluyendo su propuesta de clasificación final, debidamente justificada. Esta propuesta será discutida con los respectivos supervisores a posteriori.

Dada su extensión, hemos optado por presentar, en la Tabla 1, una parte del referencial de evaluación, para facilitar su comprensión en este texto, la versión integral aparece en el Anexo.

\section{Evaluación y conclusiones}

Este proceso evaluativo de la actitud ético-profesional del alumnado en prácticas es significativamente valorado por todos los implicados en las encuestas anuales sobre los puntos fuertes y débiles del máster, reconociéndole el mérito de orientar a los alumnos 
al éxito en la asignatura en general y, en particular, en el desarrollo de las competencias ético-profesionales.

Tabla 1.

Referencial de Evaluación de la Aptitud Ético-Profesional de Estudiantes en Prácticas

\begin{tabular}{|c|c|c|c|}
\hline Competencias & $\begin{array}{l}\text { Maestría } \\
\text { (18-20 puntos) }\end{array}$ & $\begin{array}{c}\text { Habilidad } \\
\text { (17-14 puntos) }\end{array}$ & $\begin{array}{l}\text { Aprendizaje } \\
\text { (13-10 puntos) }\end{array}$ \\
\hline $\begin{array}{l}\text { A. } \\
\text { Compromiso con } \\
\text { los aprendizajes } \\
\text { de los alumnos }\end{array}$ & $\begin{array}{l}\text { A3. Revela un compromiso } \\
\text { ético con los aprendizajes del } \\
\text { alumnado. Promueve la } \\
\text { diferenciación del } \\
\text { aprendizaje, asumiendo una } \\
\text { actitud inclusiva en la } \\
\text { totalidad de las clases ante los } \\
\text { diferentes alumnos y alumnas. } \\
\text { Promueve el éxito en los } \\
\text { alumnos. }\end{array}$ & $\begin{array}{l}\text { A2. Revela un } \\
\text { compromiso ético con los } \\
\text { aprendizajes del alumnado, } \\
\text { promoviendo la inclusión } \\
\text { de los diferentes alumnos y } \\
\text { alumnas de la clase. } \\
\text { Promueve el éxito del } \\
\text { alumnado en los } \\
\text { aprendizajes. }\end{array}$ & $\begin{array}{l}\text { A1. Revela un } \\
\text { compromiso ético con } \\
\text { los aprendizajes del } \\
\text { alumnado. }\end{array}$ \\
\hline B. & $\mathrm{C} 3$ & $\mathrm{C} 2$. & $\mathrm{C} 1$. \\
\hline$\ldots$ & $\ldots$ & $\ldots$ & $\ldots$ \\
\hline
\end{tabular}

A pesar de esto, este proceso aún presenta puntos de mejora, principalmente en relación a la necesidad de profundizar la comprensión, por parte de los alumnos y tutores, de algunos de los criterios de evaluación de la Dimensión Aptitud Ético-Profesional, como, por ejemplo, los que son más difíciles de controlar o de concretar, como es el caso de la Innovación de las prácticas pedagógicas y documentales, o el de la Autoformación y desarrollo profesional, entre otros.

\section{Referencias}

Crisol, M., \& Romero, M.A. (2014). "Práctica docente versus ética docente". Hacia la mejora de la práctica docente a partir de la ética profesional. Journal for Educators, Teachers and Trainers, 5(2), 23-35.

Decreto-Lei n ${ }^{\circ}$ 240/2001, Governo de Portugal, acedido en 17 de abril:

https://dre.pt/pesquisa//search/631837/details/normal?q=decreto+lei+240\%2F2001

Ribeiro-Silva, E., Fachada, M., \& Nobre, P. (2018). Prática pedagógica supervisionada II. Universidade de Coimbra, pp. 65-66. 


\section{Anexo}

\section{NIVELES DE EVALUACIÓN DE LA APTITUD ÉTICO-PROFESIONAL}

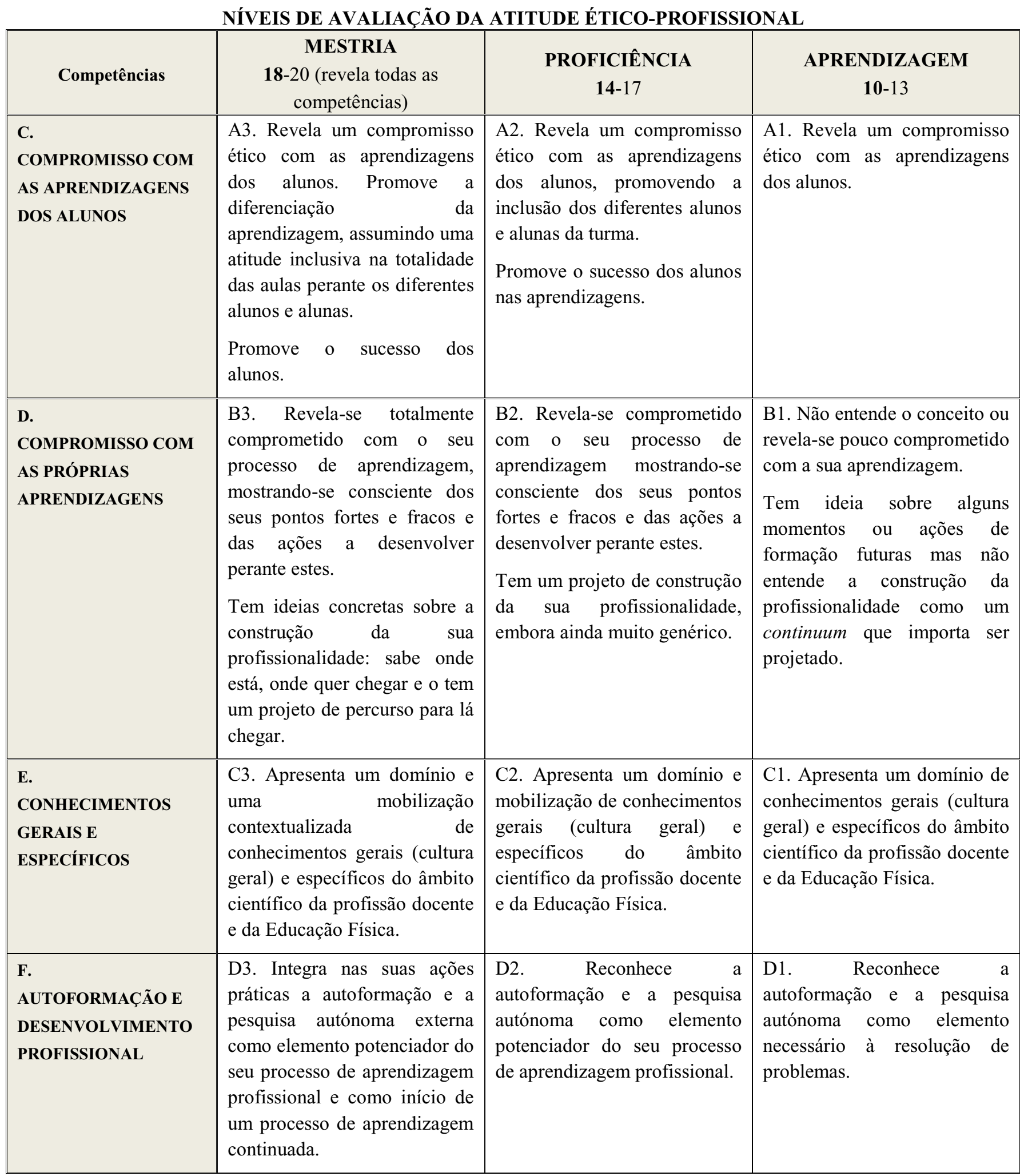




\begin{tabular}{|c|c|c|c|}
\hline $\begin{array}{l}\text { G. } \\
\text { DISPONIBILIDADE } \\
\text { PARA OS ALUNOS E } \\
\text { PARA A ESCOLA }\end{array}$ & $\begin{array}{l}\text { E3. Revela uma } \\
\text { disponibilidade sistemática } \\
\text { para os alunos e para a escola } \\
\text { com uma interação e } \\
\text { intervenção empenhada e } \\
\text { construtiva. }\end{array}$ & $\begin{array}{l}\text { E2. Revela uma } \\
\text { disponibilidade regular para os } \\
\text { alunos e para a escola, } \\
\text { procurando interagir e intervir. }\end{array}$ & $\begin{array}{l}\text { E1. Revela uma } \\
\text { disponibilidade mínima para } \\
\text { os alunos e para a escola, } \\
\text { limitando-se a cumprir os } \\
\text { requisitos mínimos (ver D.). }\end{array}$ \\
\hline $\begin{array}{l}\text { H. } \\
\text { TRABALHO EM } \\
\text { EQUIPA }\end{array}$ & $\begin{array}{l}\text { F3. Promove o trabalho de } \\
\text { equipa. Usa-o quando as } \\
\text { condições o exigem, de acordo } \\
\text { com os propósitos e de modo } \\
\text { construtivo. Anima as inter- } \\
\text { relações entre os participantes } \\
\text { e o respeito mútuo. Assume o } \\
\text { trabalho em equipa como uma } \\
\text { responsabilidade própria e } \\
\text { coletiva. }\end{array}$ & $\begin{array}{l}\text { F2. Trabalha em equipa de } \\
\text { modo construtivo. Assume o } \\
\text { trabalho de equipa como uma } \\
\text { responsabilidade própria e } \\
\text { coletiva. }\end{array}$ & $\begin{array}{l}\text { F1. Revela disponibilidade e } \\
\text { trabalha em equipa. }\end{array}$ \\
\hline $\begin{array}{l}\text { I. } \\
\text { CAPACIDADE DE } \\
\text { INICIATIVA E } \\
\text { RESPONSABILIDADE }\end{array}$ & $\begin{array}{l}\text { G3. Revela sentido de } \\
\text { responsabilidade, respeito } \\
\text { pelos compromissos } \\
\text { assumidos e capacidade de } \\
\text { iniciativa. Cumpre as } \\
\text { exigências inerentes à escola e } \\
\text { ao estágio. É assertivo e } \\
\text { assume e justifica a sua } \\
\text { responsabilidade no trabalho } \\
\text { individual e coletivo. }\end{array}$ & $\begin{array}{l}\text { G2. Revela sentido de } \\
\text { responsabilidade, respeito } \\
\text { pelos compromissos } \\
\text { assumidos e capacidade de } \\
\text { iniciativa. Cumpre as } \\
\text { exigências inerentes à escola e } \\
\text { ao estágio. Assume a sua } \\
\text { responsabilidade no trabalho } \\
\text { individual e coletivo }\end{array}$ & $\begin{array}{l}\text { G1. Revela sentido de } \\
\text { responsabilidade, respeito } \\
\text { pelos } \\
\text { assumidos e compromissos } \\
\text { iniciativa. capacidade de } \\
\text { exigências inerentes à escola e } \\
\text { ao estágio. }\end{array}$ \\
\hline $\begin{array}{l}\text { J. } \\
\text { INOVAÇÃO DAS } \\
\text { PRÁTICAS } \\
\text { PEDAGÓGICAS E } \\
\text { DOCUMENTAL }\end{array}$ & $\begin{array}{l}\text { H3. A sua intervenção } \\
\text { pedagógica é original e } \\
\text { inovadora no planeamento, na } \\
\text { realização e na reflexão sobre } \\
\text { as aulas, na produção de } \\
\text { documentos e na conceção de } \\
\text { projetos. }\end{array}$ & $\begin{array}{l}\text { H2. Apresenta } \\
\text { intervenção pedagógica } \\
\text { inovaçóes nos domínios do } \\
\text { planeamento, realização e } \\
\text { reflexão sobre as aulas e na } \\
\text { produção de documentos ou } \\
\text { conceção de projetos. }\end{array}$ & $\begin{array}{l}\text { H1. Reconhece a importância } \\
\text { da inovação relativamente às } \\
\text { suas práticas pedagógicas - no } \\
\text { planeamento, na realização e } \\
\text { na reflexão sobre as aulas. }\end{array}$ \\
\hline $\begin{array}{l}\text { K. } \\
\text { ANÁLISE CRÍTICA E } \\
\text { REFLEXIVA }\end{array}$ & $\begin{array}{l}\text { I3. Crítica e reflete de forma } \\
\text { autónoma e propõe soluções } \\
\text { credíveis para os problemas. } \\
\text { Autoavalia o seu desempenho } \\
\text { com base em referências } \\
\text { internas e externas. }\end{array}$ & $\begin{array}{l}\text { I2. Apresenta capacidade de } \\
\text { análise crítica e reflexiva. } \\
\text { Autoavalia o seu desempenho } \\
\text { com base em referências } \\
\text { externas. }\end{array}$ & $\begin{array}{l}\text { I1. Apresenta capacidade de } \\
\text { análise crítica quando } \\
\text { orientado. }\end{array}$ \\
\hline $\begin{array}{l}\text { L. } \\
\text { CONDUTA PESSOAL }\end{array}$ & $\begin{array}{l}\text { J3. Assume uma apresentação } \\
\text { e conduta pessoal adequadas } \\
\text { perante os alunos, professores } \\
\text { e funcionários; é assíduo e } \\
\text { pontual, promovendo estes } \\
\text { valores junto dos alunos e dos } \\
\text { elementos do grupo de } \\
\text { estágio. }\end{array}$ & $\begin{array}{l}\text { J2. Assume uma apresentação } \\
\text { e conduta pessoal adequadas } \\
\text { perante os alunos, professores } \\
\text { e funcionários; é assíduo e } \\
\text { pontual, promovendo estes } \\
\text { valores junto dos alunos. }\end{array}$ & $\begin{array}{l}\text { J1. Assume uma apresentação } \\
\text { e conduta pessoal adequadas } \\
\text { perante os alunos, professores } \\
\text { e funcionários; é assíduo e } \\
\text { pontual. }\end{array}$ \\
\hline
\end{tabular}

